\title{
High dimensional characterization of the longitudinal phase space formed in a radio frequency quadrupole
}

\author{
K. Ruisard๑, ${ }^{*}$ A. Aleksandrov®, S. Cousineau, V. Tzoganis๑, and A. Zhukov® \\ Oak Ridge National Laboratory, Oak Ridge, Tennessee 37830, USA
}

(Received 6 July 2020; accepted 26 October 2020; published 8 December 2020)

\begin{abstract}
Modern accelerator front ends almost exclusively include radio-frequency quadrupoles for initial capture and focusing of the low-energy beam. Dynamics in the RFQ define the longitudinal bunch parameters. Simulation of the SNS RFQ with PARMTEQ seeded with a realistic LEBT distribution produces a $2.5 \mathrm{MeV}, 40 \mathrm{~mA} \mathrm{H}$ - beam with root-mean-square emittance of $130 \mathrm{deg} \mathrm{keV}$. In measurement, a detailed characterization of the longitudinal phase space is made, including a novel study of the dependence of longitudinal emittance on transverse coordinates. This work introduces a new virtual slit technique that provides subslit resolution in an energy spectrometer as well as an approach for visualizing 4D phase space data. Through simulation and measurement, the RFQ-formed bunch is confirmed to have significant internal correlated structure. The high-dimensional features are shown to be in qualitative agreement. However, the measured rms emittances are up to $30 \%$ lower than predicted, closer to the design value of $95 \mathrm{deg} \mathrm{keV}$.
\end{abstract}

DOI: 10.1103/PhysRevAccelBeams.23.124201

\section{INTRODUCTION}

Low-level beam loss is a fact of life in high-intensity accelerator facilities. Controlling and reducing losses to maintain a safe accelerator environment is achieved mainly through online empirical optimization. One tool currently missing from the arsenal is high fidelity simulation capable of predicting these losses. As high-intensity accelerators continue toward higher demands in beam power, the need for this capability grows [1]. A strong contributor for losses in a linear accelerator is beam halo [2,3]. As the beam distribution is both the source and driver of halo particles, loss-level simulation accuracy will require an equally accurate representation of the initial distribution.

There are two approaches for generating a realistic front-end initial distribution. One is an pure "end-toend" approach, which applies self-consistent simulation of the entire beam transport system starting at or downstream of the ion source (e.g., [4]). This may include selfconsistent modeling of the ion source/extraction electrodes, abstracting to an idealized distribution in the low energy beam transport (LEBT) section, or measuring the transverse phase space in the LEBT. The LEBT distribution is then propagated through the radio frequency quadrupole (RFQ).

\footnotetext{
*ruisardkj@ornl.gov
}

Published by the American Physical Society under the terms of the Creative Commons Attribution 4.0 International license. Further distribution of this work must maintain attribution to the author(s) and the published article's title, journal citation, and DOI.
The longitudinal bunch is formed inside the RFQ, where dynamics are complicated by nonlinear focusing from both the vane structure and space charge. The complexity of the RFQ simulation may limit the accuracy of output bunch, as the effects of small differences between design and as-built/ operational machine may be amplified. Previous work at Los Alamos [5,6], found that the bunch generated through simulation of the RFQ was not sufficiently accurate to model beam dynamics in a medium-energy transport line (MEBT). Particularly, transport through mismatched optics was seen to be very sensitive to the initial distribution.

Alternatively, a bunch may be generated from measurements in the MEBT, after the longitudinal bunch is fully formed but at an energy where detailed measurements are still possible. Characterization of the beam in the MEBT circumvents the need to model the complex internal RFQ dynamics. However, internal correlations are neglected in this approach, which typically reconstructs the bunch on the basis of 2D phase space projections or rms Twiss parameters (for example, see $[7,8]$ ). Direct measurement of the 6D beam distribution has been demonstrated [9], but at present end-to-end simulation remains the most accessible option for generating fullycorrelated particle coordinates.

The formation of the longitudinal phase space is mediated by the space charge force, which couples the three planes [9-12]. As will be shown, the bunch formed in the RFQ has nontrivial internal structure; both the bunch shape and energy profile vary with distance from the high-density core. As core mismatch is known to excite halo growth 
$[3,13]$, it is reasonable to expect that loss-predictive simulations will require complete 6D distributions.

Given that end-to-end simulation is the most readily available source of fully correlated bunches, one may wonder to what extent simulation reproduces the full $6 \mathrm{D}$ structure. If there is discrepancy in the rms predictions, can one still trust the high-dimensional features? To begin addressing this question, a detailed characterization of longitudinal phase space is compared with predictions from RFQ simulation. While the primary metric is the rms emittance, it is applied to slice emittances rather than full emittance. By varying slice dimensionality and location, the dependence of longitudinal emittance on transverse coordinates is studied. This provides a method to visualize the high-dimensional features inside the RFQ bunch.

\section{A. SNS beam test facility}

The SNS beam test facility (BTF) is a one-to-one replica of the SNS front-end, composed of $50 \mathrm{~mA} \mathrm{H}$ - ion source, $65 \mathrm{kV}$ LEBT, $402.5 \mathrm{MHz}$ RFQ and 1.3 meters of MEBT quadrupoles. In addition, the BTF is equipped with extensive diagnostics enabling direct measurement of the 6D phase space distribution. This phase space diagnostic includes two pairs of vertical/horizontal slits for selection of transverse phase space coordinates, followed by an energy spectrometer comprised of a $90^{\circ}$ dipole and vertical slit. Finally, a bunch shape monitor (BSM) $[14,15]$ records the phase profile as sampled through time-of-arrival of secondary electron emission from a beam-intersecting wire. The energy spectrometer and bunch shape monitor are used to reconstruct the longitudinal phase space.

Accelerator physics studies at the BTF are motivated by the goal of demonstrating halo-predictive simulation. Ongoing efforts have followed a three-pronged approach: extensive characterization of the initial MEBT beam distribution [9], deployment of high dynamic range phase space diagnostics for halo detection, and extension of the BTF MEBT to support studies of halo evolution [16,17]. The work described here falls in the first category, as it explores the fidelity of modeling RFQ bunch formation.

\section{B. Emittance convention}

The rms emittance is used to quantify the longitudinal phase space. The coordinates for longitudinal position and momentum are phase $\phi$, with units rf degrees, and energy $w$, with units $\mathrm{keV}$. Rms emittance $\epsilon_{z}$ is defined as

$$
\epsilon_{z}=\sqrt{\left\langle\phi^{2}\right\rangle\left\langle w^{2}\right\rangle-\langle\phi w\rangle^{2}} .
$$

For a density function $f(\phi, w)$,

$$
\left\langle\phi^{m} w^{n}\right\rangle=\frac{\int_{-\infty}^{+\infty} d \phi d w \phi^{m} w^{n} f(\phi, w)}{\int_{-\infty}^{+\infty} d \phi d w f(\phi, w)} .
$$

The rms Twiss parameters $\beta$ and $\alpha$ are defined following the convention $\left\langle\phi^{2}\right\rangle=\beta \epsilon$ and $\langle\phi w\rangle=-\alpha \epsilon$. This article reports transverse emittances in energy-normalized coordinates, following the typical convention of $\epsilon_{x, n o r m .}=\beta \gamma \epsilon_{x}$ for relativistic $\beta, \gamma$. Longitudinal emittances are reported unnormalized.

As the rms parameters can depend heavily on the threshold, it is necessary to specify the threshold applied to $f(\phi, w)$. We adopt the following metrics for reporting emittances: (i) $0.1 \%$ emittance, near dynamic-range limit of measurements, (ii) $1 \%$ emittance, and (iii) $10 \%$ emittance, representing the core of the beam. Thresholds are defined with respect to peak density. In simulation it is common to report emittances based on percentage of enclosed particles. This is distinct from the definitions here. " $100 \%$ emittance" is used in the usual sense to indicate inclusion of all simulation particles.

In plots of the longitudinal phase space, positive phase corresponds to positive time. The tail of the bunch, which arrives at a later time than the head, has $\phi_{\text {tail }}>\phi_{\text {head }}$.

\section{Dimensionality}

In addition to threshold, the reported emittances will depend on the dimensionality of the phase space used for the calculation. Typically, emittances are calculated for the fully projected phase space. That is, the $(\phi, w)$ coordinates of every particle regardless of location in transverse phase space are included. The BTF longitudinal emittance apparatus samples phase space for a 3D slice in the transverse coordinates. This is a partially projected phase space, and the resulting emittance a partial or slice emittance.

It should be immediately clear that many unique partial projections of the longitudinal phase space are possible. Slices can be made in one or several or all of the transverse coordinates, and the slices can be taken at varying distance from the beam core. Figure 1 illustrates the slice concept in three dimensions. As slices are generally made in hidden (unplotted) dimensions, it is not explicitly apparent whether

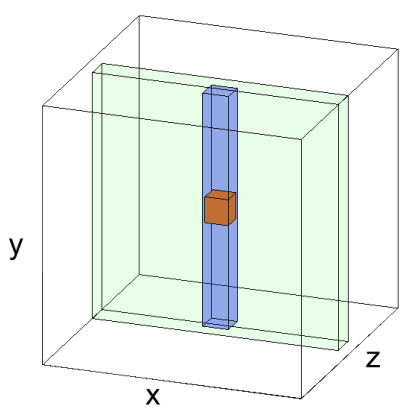

FIG. 1. Illustration of high dimensional slices in 3D space. A slice of the cube can be made along one dimension (e.g., $\tilde{z}=0$, the green volume), two dimensions $(\tilde{z}, \tilde{x}=0$, blue) or all three dimensions $(\tilde{z}, \tilde{x}, \tilde{y}=0$, red). If the $1 \mathrm{D}$ and $2 \mathrm{D}$ slice volumes are projected onto the $y, z$ plane, they will be indistinguishable, as the slice $\tilde{x}$ is along an unplotted dimension. 
a phase space plot represents a full or n-dimensional partial projection. Therefore, when discussing slice emittances, it is important to indicate both the dimensionality (number of slices) and each slice location.

Here, $f$ indicates the full $6 \mathrm{D}$ phase space density $f\left(x, x^{\prime}, y, y^{\prime}, \phi, w\right) . \hat{f}$ indicates a partial projection, where a finite-width slice is made in at least one dimension. The dimensions are listed as subscripts, for example $\left.\hat{f}\right|_{\tilde{x}, \tilde{y}}$ for a 2D $x, y$ slice. The tilde indicates a finite-width slice, equal to the physical apertures unless otherwise specified. If coordinates do not appear as arguments or slices of $\hat{f}$, the density is integrated along that axis without slicing. With this notation, the longitudinal phase space for particles within a $2 \mathrm{D}$ slice centered at $x=0, x^{\prime}=0$ is indicated as $\left.\hat{f}(\phi, w)\right|_{\tilde{x}, \tilde{x}^{\prime}=0}$.

The organization of this article is as follows. Section II defines the "expected distribution", as determined through RFQ simulation. After that, the measurement technique is introduced in Sec. III, including accounting for the dominant error in phase width via application of the virtual slit method. The high dimensional characterization of longitudinal phase space is reported in Sec. IV. The results show the dependence of the longitudinal slice distribution on RFQ amplitude and transverse coordinates. Finally, Sec. V summarizes the comparison between the expected and measured distributions.

\section{SIMULATION}

\section{A. RFQ output distribution}

Original design studies for the SNS RFQ used the Los Alamos code PARMTEQ [18]. The RFQ accelerates $H^{-}$from $65 \mathrm{kV}$ to $2.5 \mathrm{MeV}$, achieved with vane voltage $83 \mathrm{kV}$ and 449 cells. Additionally, the design is constrained to produce $\leq 95$ $\mathrm{keV}$-deg at maximum current output. This goal was met with normalized input emittance $0.2 \mathrm{~mm}$-mrad and simulation transmission $>90 \%$ [19-21]. In this paper, the PARMTEQ simulation is repeated with an input beam based on LEBT measurements, using the most recent version PARMTEQM.
The PARMTEQ space charge calculation uses the SCHEFF module with a cylindrical geometry. Saturation was judged by the rms Twiss parameters of the output bunch. 40,000 particles and a grid spacing of 10 radial segments and 20 longitudinal segments was sufficient. However, for the results reported here up to 5,000,000 macroparticles are used. The higher particle number was necessary for good statistics when calculating rms emittances for high-dimensional slices.

The input beam is initially mono-energetic with $w \equiv$ $T-T_{0}=0$ for all particles, and initial uniform random phase. The transverse distribution is generated from measurements of the horizontal and vertical phase space distributions in the LEBT. These measurements were acquired in 2012 with ion source output of $50 \mathrm{~mA}$.

It is assumed that the LEBT distribution is matched to the RFQ acceptance, as the LEBT optics are tuned for optimal RFQ transmission. The distribution is measured at a plane several centimeters downstream of the RFQ entrance plane. To satisfy the matched condition, the measured coordinates are transformed via beam transport matrices for optimal overlap with the acceptance ellipse. The resulting transverse phase space distributions are plotted in Figs. 2(a) and 2(b). The rms Twiss parameters are reported in Table I.

As the motivation of this study is to address the role of RFQ simulations in high-fidelity modeling, the measurement-based LEBT distribution is used to generate the expected distribution, under the assumption that this is the most likely to resemble the actual beam parameters. However, comparison to equivalent rms distributions can quantify sensitivity to initial distribution. Equivalent is defined as having identical rms Twiss parameters.

Table II reports parameters of the RFQ output distribution and compares to cases with equivalent $\mathrm{KV}$ and $4 \mathrm{D}$ waterbag distributions. These are defined as the surface of 4D ellipsoid [22] and a uniformly filled ellipsoid, respectively. Both equivalent distributions predict slightly higher transmission and lower emittance. For the realistic case, the $100 \% \mathrm{rms}$ emittance is $\epsilon_{z}=127 \mathrm{deg} \mathrm{keV}$, with fully

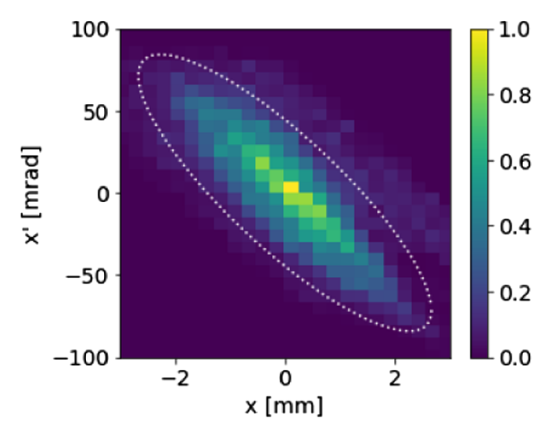

(a)

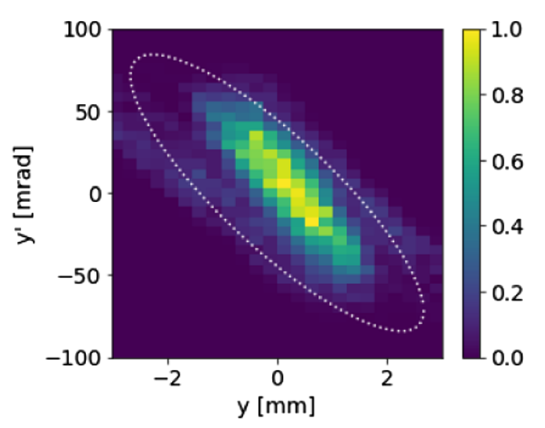

(b)

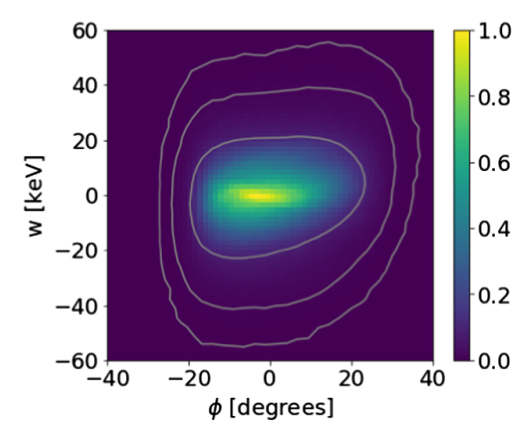

(c)

FIG. 2. Fully projected phase space plots for initial (2(a), 2(b)) and output (2(c)) distributions from PARMTEQ simulation. Density is plotted in linear scale. Dotted lines in (2(a), 2(b)) show the RFQ acceptance ellipse $\alpha=1.6, \beta=0.06$ at $\epsilon=1.4 \mathrm{~mm}$ mrad [norm.]. The contours on (2(c)) show the $10 \%, 1 \%$, and $0.1 \%$ threshold levels. 
TABLE I. $100 \%$ rms parameters of the realistic LEBT distribution at the entrance to the RFQ.

\begin{tabular}{lcc}
\hline \hline Quantity & Horizontal & Vertical \\
\hline$\epsilon$ [norm, mm-mrad] & 0.24 & 0.24 \\
$\alpha$ & 1.08 & 0.97 \\
$\beta[\mathrm{mm} / \mathrm{rad}]$ & 51 & 49 \\
\hline \hline
\end{tabular}

projected rms widths $\phi_{\text {rms }}=10.6^{\circ}$ and $w_{\text {rms }}=12.2 \mathrm{keV}$. The 2D full-projection is shown in Fig. 2(c).

Figure 3 illustrates the importance of distinguishing between slice and full emittance by plotting the dependence of the rms longitudinal emittance on the width of a transverse $\left(x, x^{\prime}\right)$ slice centered over the beam core. The narrowest slices (left-most points) have widths comparable to the measurement resolution, $x= \pm 0.1 \mathrm{~mm}$ and $x^{\prime}=$ $\pm 0.2 \mathrm{mrad}$. The output bunch formed for three different initial distributions have very complex structure compared to a Gaussian beam, which would appear as a straight horizontal line. In the waterbag and KV case, the emittance of the core slice is $10 \%$ lower than the full emittance. In the realistic case, for which the initial bunch resembles a Gaussian with heavy, nonlinear tails, the core slice is very similar to the full emittance but there are still distinct features. For all distributions, the emittance of an arbitrary slice should not be assumed to be representative of the full emittance.

\section{B. Expected distribution in the MEBT}

The BTF measurements are made with respect to a plane 1.36 meters downstream of the RFQ. The reference point is the location of the first vertical slit used in the phase space measurement. After this point, at least $98 \%$ of the beam is intercepted. In the remaining $2 \%$ "beamlet", there should be no contribution from space charge on the beam evolution, and downstream measurements can easily be mapped to this plane via matrix equations. For the purposes of comparison, it is considerably more straightforward to propagate the self-consistent 6D PARMTEQ distribution to the measurement plane than back-propagate the measured phase space. With this in mind, the expected distribution is defined as the output from PARMTEQ simulation seeded

TABLE II. rms parameters of the bunch at the RFQ output, based on PARMTEQ simulation for different input distributions. Transmission calculated for $50 \mathrm{~mA}$ input current. Longitudinal emittances are $100 \%$, unnorm.

\begin{tabular}{lccc}
\hline \hline Input distribution & Realistic & 4D Waterbag & KV \\
\hline Transmission & $82 \%$ & $90 \%$ & $88 \%$ \\
$\epsilon_{z}[\mathrm{deg} \mathrm{keV}]$ & 127 & 89 & 102 \\
$\alpha_{z}$ & 0.18 & 0.27 & 0.21 \\
$\beta_{z}[\mathrm{deg} / \mathrm{keV}]$ & 0.88 & 1.38 & 1.16 \\
$\epsilon_{x}, \epsilon_{y}[\mathrm{norm}, \mathrm{mm}-\mathrm{mrad}]$ & 0.22 & 0.12 & 0.15 \\
\hline \hline
\end{tabular}

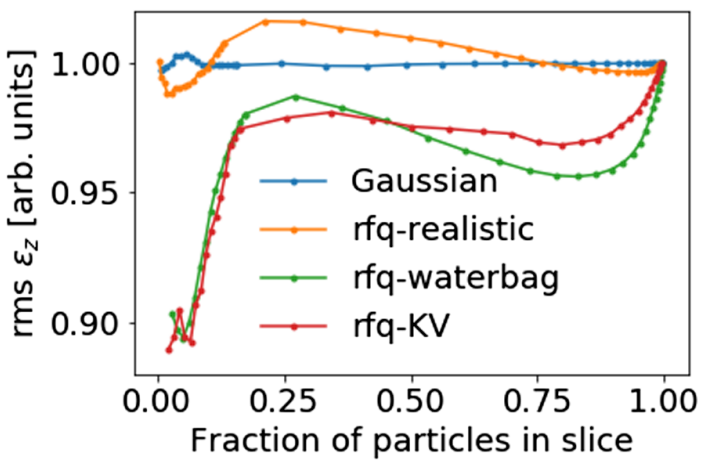

FIG. 3. Dependence of calculated longitudinal emittance on width of the slice in coordinates $\left(x, x^{\prime}\right)$ centered at $(0,0)$. Width is expressed as fraction of particles that fall within the slice. Curves are normalized to the $100 \%$ emittances reported in Table II. The 6D Gaussian illustrates the effect of particle noise in the narrowest slices.

with the initial measured LEBT distribution at the plane of the first slit in the emittance apparatus.

Modeling of the MEBT is done with the particle-in-cell code PyORBIT [23]. Between the RFQ and the first slit, the MEBT contains four quadrupoles. A hard-edged model was used, with parameters listed in Table III. A stepsize of $1 \mathrm{~cm}$ is used for the space charge calculation.

The expected distribution at the measurement plane is plotted in Fig. 4. For the fully projected phase space, the $100 \%$ rms emittance is $\epsilon_{z}=131 \mathrm{deg} \mathrm{keV}$ and the rms widths are $5.6^{\circ}$ and $24 \mathrm{keV}$. The phase width is reported for the "shear-corrected" frame, where the linear phase correlation has been subtracted. For comparison, the uncorrected phase width at this location is $43^{\circ}$. The bunch has significantly different aspect ratio than at the RFQ exit $\left(10.6^{\circ}\right.$ and $\left.12.2 \mathrm{keV}\right)$ due to space charge driven debunching in the first $20 \mathrm{~cm}$ of transport [24]. This causes the energy spread to increase by $\sim 2 \times$ and the phase width to decrease by $\sim 2 \times$.

Table IV compares the 1\%-thresholded emittance and rms widths for slices of different dimensionality in the expected distribution. The rms emittance does not have a strong dependence on the dimensionality of the slice. In the context of measurement, the variation in emittance values is comparable to the uncertainty in measurement, which will be shown to be around $10 \%-15 \%$. As such, the measured

TABLE III. Parameters used for four-quadrupole MEBT transport line between RFQ at $s=0$ and plane of first vertical slit at $s=1.326$.

\begin{tabular}{lccc}
\hline \hline Position $[\mathrm{m}]$ & $L_{\text {eff }}[\mathrm{m}]$ & $\int B \cdot d l[\mathrm{~T}]$ & Polarity \\
\hline 0.131 & 0.061 & 1.12 & $\mathrm{~F}$ \\
0.314 & 0.066 & -1.25 & $\mathrm{D}$ \\
0.575 & 0.096 & 1.08 & $\mathrm{~F}$ \\
0.771 & 0.096 & -0.61 & $\mathrm{D}$ \\
\hline \hline
\end{tabular}




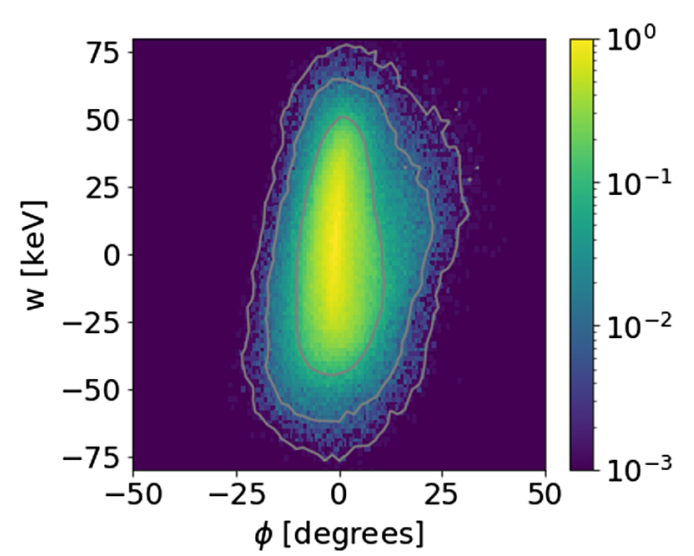

FIG. 4. Expected longitudinal phase space generated via PARMTEQ and PyORBIT propagation of the realistic LEBT distribution. Emittance is plotted at the location of the first slit in the MEBT.

values of core-slice emittances are expected to be very near the fully projected emittance. Also apparent in Table IV is that the energy/phase aspect ratio increases with dimensionality. The space charge driven debunching is amplified in the high-density core, which is preferentially included in centered, high-dimensional slices. For this reason, the rms energy spread becomes larger and the rms phase spread smaller for core slices when compared to the fully projected phase space.

Figure 5 shows the dependence of the expected energy profile $\hat{f}(w)$ on the dimensionality of a slice in transverse dimensions. This illustrates the increase of rms energy width reported in Table IV as well as the presence of very non-Gaussian internal structure. This structure strongly resembles with the initial observation of high-dimensional correlations reported in [9].

\section{APPARATUS}

The apparatus for longitudinal emittance measurement at the SNS BTF is a combination of an energy spectrometer and bunch shape monitor. This apparatus employs a system of three slits and a $90^{\circ}$ dipole to select energy upstream of a

TABLE IV. Dependence of rms quantities of expected distribution on dimensionality of phase space slice. All slices are centered over the beam core. rms values are calculated with $1 \%$ threshold applied. For these results, 5,000,000 macroparticles are used to obtain good statistics in high-dimensional slices. The slice widths are twice as large as in measurement for the same reason.

\begin{tabular}{lccc}
\hline \hline Slice & $\epsilon_{z}[\mathrm{deg} \mathrm{keV}]$ & $\mathrm{rms} \phi[\mathrm{deg}]$ & $\mathrm{rms} w[\mathrm{keV}]$ \\
\hline None (full) & 122 & 5.4 & 22.8 \\
$\tilde{y}=0$ & 117 & 5.1 & 22.9 \\
$\tilde{y}, \tilde{x}=0$ & 135 & 5.2 & 25.8 \\
$\tilde{y}, \tilde{x}, \tilde{x}^{\prime}=0$ & 128 & 4.9 & 26.4 \\
$\tilde{y}, \tilde{x}, \tilde{x}^{\prime}, \tilde{y}=0$ & 144 & 4.9 & 29.8 \\
\hline \hline
\end{tabular}

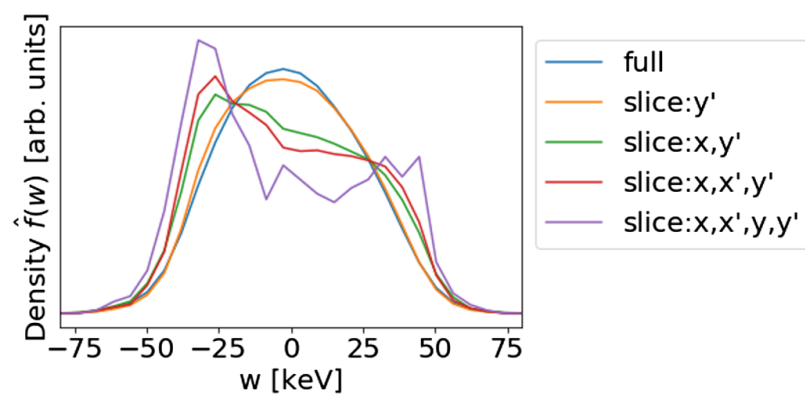

FIG. 5. Dependence of partially projected energy profile $\hat{f}(w)$ on dimensionality of slice.

bunch shape monitor that images the phase distribution. The apparatus is described in more detail in [9], where it was used to measure the full $6 \mathrm{D}$ distribution.

Figure 6 shows a diagram of the apparatus overlaid on a plot of the transverse optics. Energy selection is made with a vertical slit downstream of the $90^{\circ}$ dipole. Upstream of the dipole, two vertical slits isolate $x$ and $x^{\prime}$ coordinates to create a collimated beam at the dipole entrance. All slits are designed to have the same width. The width of the third slit is measured to be $0.17 \pm 0.02 \mathrm{~mm}$. The selected energy is a function of the location of all three slits and is calculated via matrix transformation.

After energy is selected, the beamlet travels through an additional two quadrupoles to the bunch shape monitor (BSM). The BSM is comprised of a $200 \mu$ m-wide horizontal wire that intersects the beam. This wire emits secondary electrons, which are collected and focused onto a microchannel plate. Between the wire and plate, an $\mathrm{rf}$ deflecting field streaks the beam so that vertical position at the plate corresponds to time-of-arrival. The microchannel plate amplifies the electron signal which is then imaged via a phosphor screen and camera. The camera images the partial phase distribution $\left.\hat{f}(\phi)\right|_{\tilde{w}, \tilde{x}, \tilde{x}^{\prime}, \tilde{y}_{2}}$ for the fraction of beam selected by the three vertical slits and the BSM wire. Thanks to the sensitivity of the BSM screen and the high bit depth of the BSM camera, a signal-to-noise ratio of $10^{3.22}$ was achieved.

By varying the energy selected by the third slit, the partial phase space $\hat{f}(\phi, w)$ can be reconstructed. An example $\hat{f}(\phi, w)$ near peak density is shown in Fig. 7 . The phase space ellipse is upright in a shear-corrected frame from which the linear $\phi w$ correlation is subtracted.

\section{A. Dimensionality of measurement}

Due to selection by upstream slits and the intersection of the horizontal BSM wire, the measured $\hat{f}(\phi, w)$ phase space is a partial projection that samples from a small fraction of the total phase space volume. The partial projection measured with BTF longitudinal emittance apparatus is: 


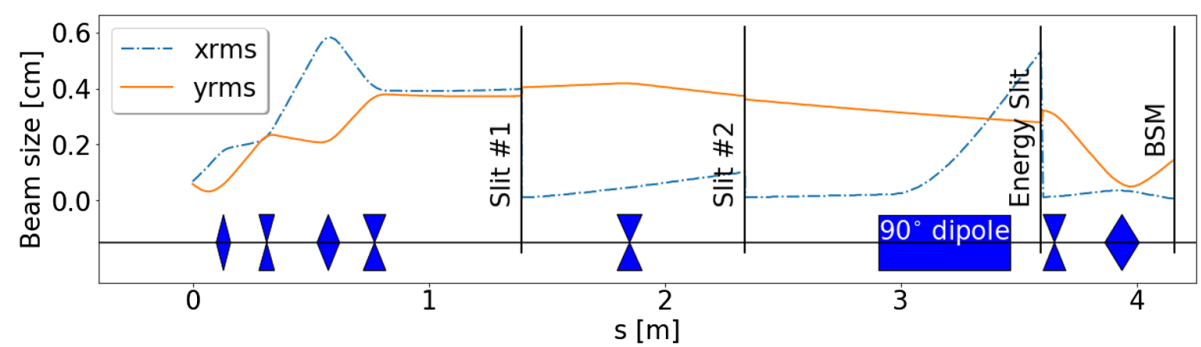

FIG. 6. Optics view of longitudinal emittance measurement, showing the location and effect of the three vertical slits and bunch shape monitor (BSM). Position $s$ is measured from the exit face of the RFQ. After Slit \#1, the scale of the x-rms curve is mm, rather than cm.

$$
\hat{f}(\phi, w)=\left.\int d y_{1} f\left(x, x^{\prime}, y_{1}, y_{2}, \phi, w\right)\right|_{\tilde{x}, \tilde{x}^{\prime}, \tilde{y}_{2}}
$$

where $\tilde{x}=x_{0} \pm \Delta x, \tilde{x}^{\prime}=x_{0}^{\prime} \pm \Delta x^{\prime}, \tilde{y}_{2}=y_{2,0} \pm \Delta y_{2}$.

Notice that the vertical coordinates are in a frame $y_{1}, y_{2}$ rather than the standard $y, y^{\prime}$. At the BSM location, the vertical slice that is selected is $\tilde{y}=y_{\text {wire }} \pm \Delta y$. However, the reference plane (first vertical slit) is upstream of the BSM wire by 2.2 meters, including four quadrupoles and one $90^{\circ}$ dipole. The slice made by the BSM wire is rotated in the $y, y^{\prime}$ phase space at the reference plane.

Figure 8 shows the vertical phase space of the beam at the first slit location, measured using a slit-scan approach. The shadow of the BSM wire is visible. The phase advance between the first slit and BSM is calculated to be approximately $100^{\circ}$. This is inferred from the vertical rms parameters at the first slit and the orientation of the wire shadow. Phase advance of less than $360^{\circ}$ is supported by simulation.

The most complete description of the beam distribution is obtained through 6D scans, as demonstrated in [9]. However, interrogating a high-dimensional volume incurs a penalty of exponentially-increased measurement time, which can only be balanced with reduction in number or density of points. For the longitudinal emittance, 2D phase

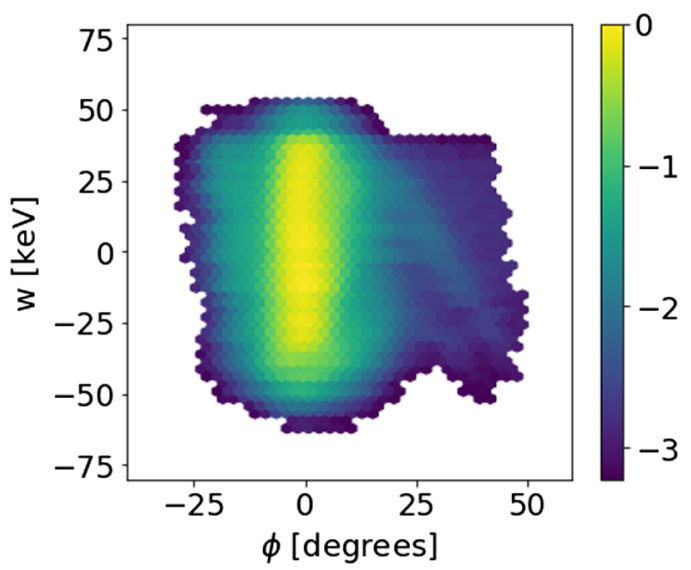

FIG. 7. Measured phase space partial projection near the beam center, $\left.\hat{f}(\phi, w)\right|_{\tilde{x}, \tilde{x}^{\prime}, \tilde{y}_{2} \sim 0}$. A threshold is applied at $6 \times 10^{-4}$ of the peak density. space is reconstructed for a 3D transverse slice (only one transverse dimension, $y_{1}$, is projected during the measurement). Sampling the entire beam requires a scan over 5D, but this would have a very long duration for the desired dynamics range and resolution: 9 days of continuous measurement would be required to cover a grid of size $14 \times 14 \times 14 \times 40$ in $\left(y_{2}, x, x^{\prime}, w\right)$ with averaging of 20 shots/point. As an alternative, this paper utilizes data from 4D scans, where the 4D scan iterates through coordinates $\left(x, x^{\prime}, w\right)$ and instantaneously images $\hat{f}(\phi)$. Each scan requires 16 hours. The dependence on the fifth coordinate, $y_{2}$, is explored by repeating the 4D scan over a range of BSM wire positions.

\section{B. Accounting for point-spread increase to measured phase}

As noted in Sec. II B, space charge defocusing causes a narrowing in phase downstream of the RFQ. This brings the phase width close to the phase resolution of the measurement. The phase resolution is not limited by the resolution of the BSM, but by the point spread function originating from the finite slit widths.

Point spread is a systematic, asymmetric error that acts to inflate the measured rms values. While the total point-

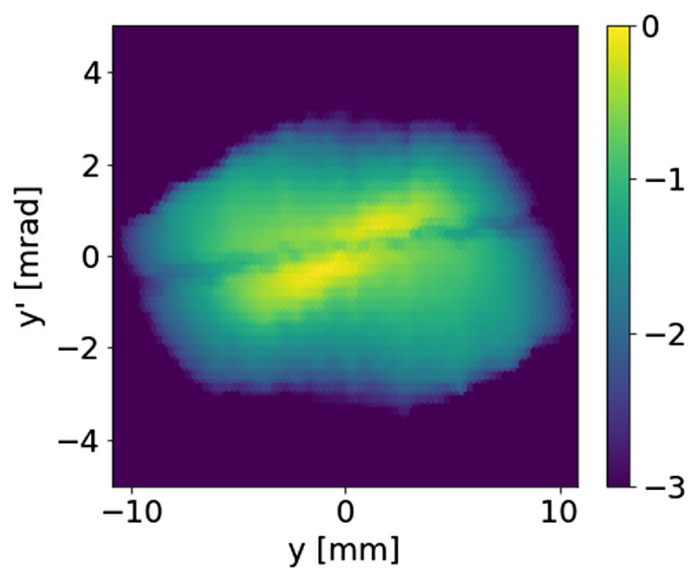

FIG. 8. Vertical phase space at the first slit with logarithmic intensity, showing the BSM wire bisecting the beam core. At $1 \%$ threshold, the vetrical $\mathrm{rms}$ parameters are $\alpha=-0.07$, $\beta=3.7 \mathrm{~m} / \mathrm{rad}$. 
TABLE V. Values for rms point-spread function and $1 \sigma$ error bars.

\begin{tabular}{lcc}
\hline \hline Quantity & $w[\mathrm{keV}]$ & $\phi\left[^{\circ}\right]$ \\
\hline Uncertainty & 0.4 & 0.6 \\
Point-spread & 0.6 & 3.3 \\
\hline \hline
\end{tabular}

spread is the combination of the three vertical slits, BSM wire and internal BSM electron focusing, the dominant contribution is the width of the third (energy) slit, which affects measurement of both phase and energy. Table $\mathrm{V}$ summarizes the rms point spread widths, as well as systematic uncertainty originating primarily from uncertainty in calibration curves. As seen in Table V, the rms phase point-spread is much larger than the uncertainty, and comparable to the expected rms width $5.6^{\circ}$. The origin and calculation of errors are discussed in more detail in the Appendix A.

The majority of the $3.3^{\circ}$ point-spread is due to the large $\phi-w$ correlation at the BSM plane. For the selected energy slice, the measured phase profile will be wider than the monoenergetic profile. In comparison, the effect of the energy point-spread is negligible, as the estimated $0.6 \mathrm{keV}$ rms energy spread selected by the $0.2 \mathrm{~mm}$ slit is much smaller than the $23 \mathrm{keV}$ expected width. While future improvement may be possible through installation of a narrower slit, given the present limitations of the apparatus it is necessary to estimate the correction to the point spread on the measured rms phase width.

For a Gaussian distribution and point-spread function, the inflated rms values can be corrected through subtraction in quadrature, e.g., $\left\langle\phi^{2}\right\rangle=\left\langle\phi_{\text {meas }}^{2}\right\rangle-\left\langle\phi_{\text {p.s.f. }}^{2}\right\rangle$. However, both the expected distribution described in Sec. II B and the point-spread function have significantly non-Gaussian features. Therefore, in this analysis correction to the rms values is estimated on the basis of simulated and measured recovery of the "true" phase width, which suggest a much smaller correction than estimated through propagation of Gaussian errors.

In simulation, the point spread contribution is estimated by propagating the expected distribution through a PyORBIT model of the three-slit longitudinal phase space apparatus shown in Fig. 6. Details of the approach are in Appendix B.

In measurement, it is possible to obtain subslit phase resolution through application of a novel virtual slit method. The virtual slit method requires collecting two phase profiles separated by a differential step in energy slit position and subtracting one from the other. The difference waveform includes peak and an antipeak aligned with the leading and trailing edges of the phase profile, as illustrated in Fig. 9. The difference profiles correspond to the phase profile of a beamlet selected by a virtual slit of width equal to the step size. The technique is analogous to the use of scrapers in beam profile measurements, in which

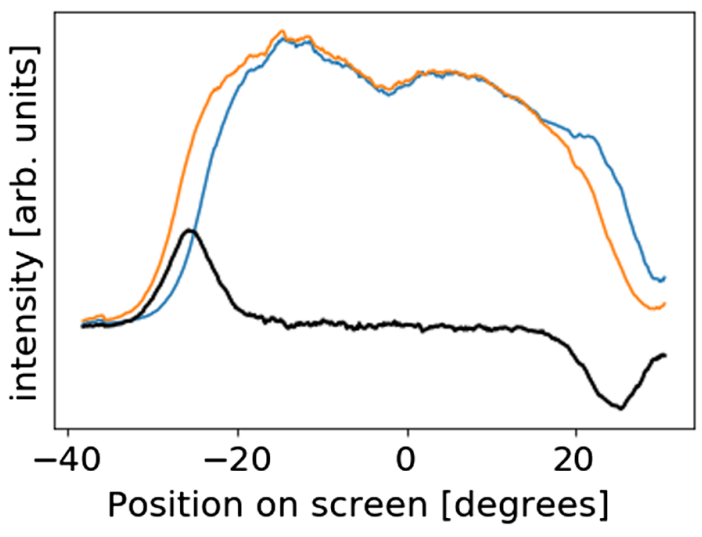

FIG. 9. Illustration of virtual slit concept. Two phase profiles (thin curves) are measured with the BSM camera for two dipole magnet settings separated by $0.05 \mathrm{~A}$. The profiles plotted are obtained with a much wider slit $(1 \mathrm{~mm})$ than the standard $0.2 \mathrm{~mm}$ slit used for emittance measurements, and the phase profile nearly fills the camera frame. The heavy black line is the differential profile, which recovers two narrower profiles corresponding with the two edges of the wide profile.

transmission is measured as a function of scraper position and differentiated to recover the spatial profile. As the technique doubles data collection time and reduces dynamic range, it is not directly applied to the measurements reported in Sec. IV. For more details, see Appendix C and Ref. [25].

Comparison of the "true" to measured rms values in both simulated reconstruction and virtual slit measurement allows determination of an appropriate correction factor. In this case, a multiplicative correction to the rms phase, energy and emittance reduces the systematic point-spread error to well within the uncertainty interval. As expected, the point-spread function has a relatively small effect on the energy distribution: the "true" rms energy width was roughly $95 \%$ of the raw measured width in both simulation and experiment. The correction to phase width is larger, as expected. Additionally, the required correction has a threshold dependence; as more tails are included in the rms calculation, the relative point-spread error is smaller. At $1 \%$ threshold, a correction factor of $87 \%$ minimizes the residual error in simulation and experiment. At $10 \%$ threshold, the corrected value decreases to $83 \%$ of the raw width. There is not enough dynamic range in simulation or measurement to recommend a correction at the $0.1 \%$ threshold. For the analysis here, the same $87 \%$ correction factor is applied to the $0.1 \%$ threshold values.

Figure 10 illustrates the magnitude of the rms phase and emittance correction against the "true" error. The onesigma uncertainty interval is plotted as well; the uncertainty on emittance is estimated by Gaussian error propagation of Table $\mathrm{V}$ values under the assumption $\epsilon_{z} \approx \Delta \phi \Delta w$. In this article both the uncertainty and the correction factor to rms 


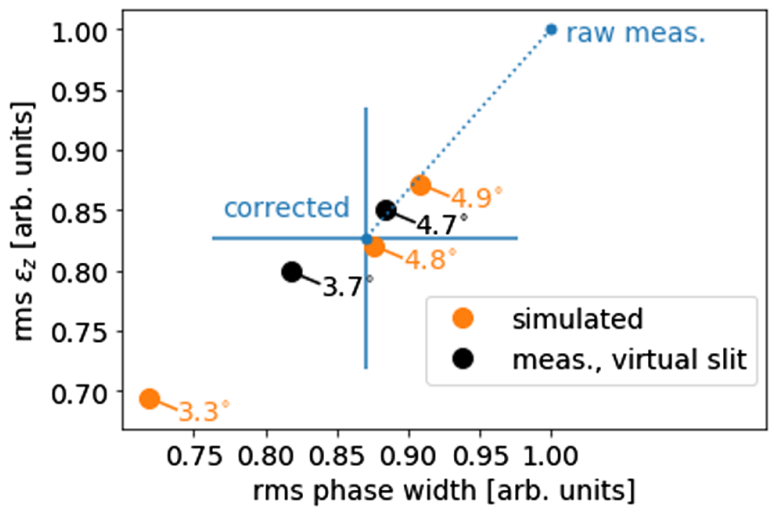

FIG. 10. Comparison of analytic error estimate with actual errors for simulated and measured emittance measurements. The scale is defined relative to the raw measurement value. The line shows the measured and corrected value for the phase space shown in Fig. 7 with $1 \sigma$ error bars on the corrected value. The scatter points show the relative values of the "true" emittance and phase widths, as determined from the virtual slit measurement and simulated emittance reconstruction.

quantities are reported explicitly in the format: raw value correction \pm uncertainty.

\section{MEASUREMENTS}

Measurements of the longitudinal emittance, which as described above is a high-dimensional slice through the transverse phase spaces, are repeated many times to map the dependence on several parameters. First, emittance is measured over a range of RFQ voltages, which is a free parameter that may be set to obtain minimum output emittance. Second, a four-dimensional scan is used to map dependence of the emittance on the transverse dimensions. This is then integrated to reconstruct the lower dimensional partial projection $\left.\hat{f}(\phi, w)\right|_{\tilde{y}_{2}}$. Finally, the $4 \mathrm{D}$ scan is repeated for several BSM wire locations, to measure dependence on the coordinate $\tilde{y}_{2}$. Measurements of the longitudinal emittance are done at nominally $20-25 \mathrm{~mA}$ average current out of the RFQ.

\section{A. Dependence on RFQ amplitude}

The longitudinal phase space is determined by the RFQ parameters. Particularly, the RFQ vane voltage may be tuned to produce the optimal (minimal) output emittance. For each voltage amplitude, the slice emittance $\left.\hat{f}(\phi, w)\right|_{\tilde{x}, \tilde{x}^{\prime}, \tilde{y}_{2}}$ is measured for a single $\tilde{x}, \tilde{x}^{\prime}, \tilde{y}_{2}$ slice centered over the peak density.

Figure 11(a) shows the result of varying RFQ amplitude on longitudinal slice emittance The raw emittance values are corrected and uncertainty assigned according to Sec. III B. Simulated values of the full emittance at the RFQ exit are included for comparison, for both the realistic LEBT and rms-equivalent waterbag initial distributions. For these simulations, 20,000 macroparticles are used.

Figure 11(b) compares simulated and measured MEBT current as a function of RFQ amplitude. For $50 \mathrm{~mA}$ LEBT current, simulations predict $41 \mathrm{~mA}$ transmitted to the MEBT. In experiment, MEBT current was significantly less. This likely due to a combination of factors, including reduced ion source current ( $40 \mathrm{~mA})$, losses in the LEBT and reduced RFQ transmission. In simulation the LEBT current is set to $23 \mathrm{~mA}$ to agree with the measured MEBT current at design voltage. The Faraday cup is located 6 meters downstream of the RFQ exit, including eleven quadrupoles and two $90^{\circ}$ dipoles, which may account for reduced transmission for low-amplitude values.

The applied vane voltage is not well-known for the test-stand RFQ. Instead, the constant of proportionality between setpoint and vane voltage is chosen for maximum overlap with the simulated curves. Relative vane voltage is defined as fraction of $83 \mathrm{kV}$ design value. The minimum measured emittance occurs at relative amplitude 0.96 , for estimated $80 \mathrm{kV}$ vane voltage. The RFQ voltage is assumed

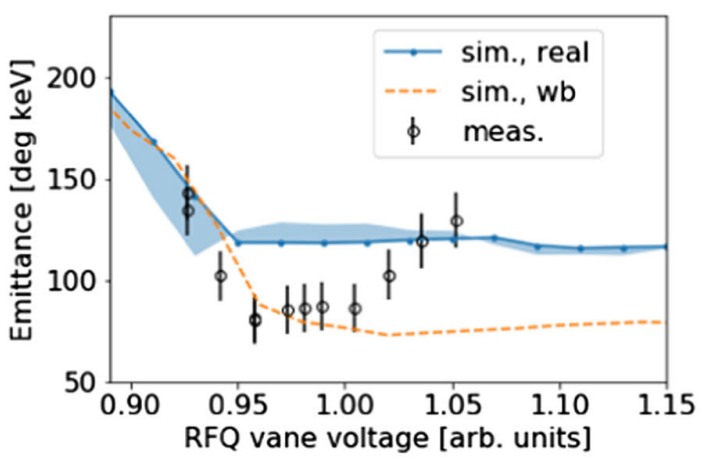

(a)

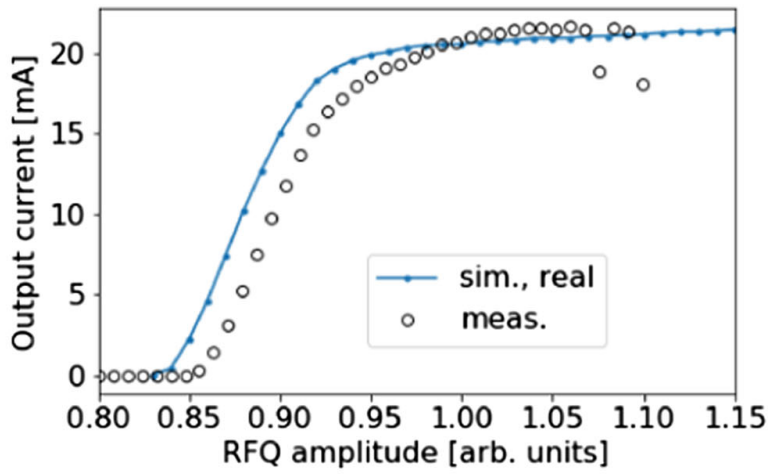

(b)

FIG. 11. Dependence of transmission and emittance on RFQ vane voltage. (a) Measured slice emittance compared to fully-projected simulation emittance with $1 \%$ thresholds. The shaded region illustrates uncertainty due to LEBT current, with boundaries at $50 \mathrm{~mA}$ and $20 \mathrm{~mA}$. The orange dashed curve shows the result for an rms-equivalent, $50 \mathrm{~mA}$ waterbag distribution. (b) Measured vs simulated transmitted current. The simulated curve has initial LEBT current $23 \mathrm{~mA}$, adjusted to agree with measured MEBT current. 


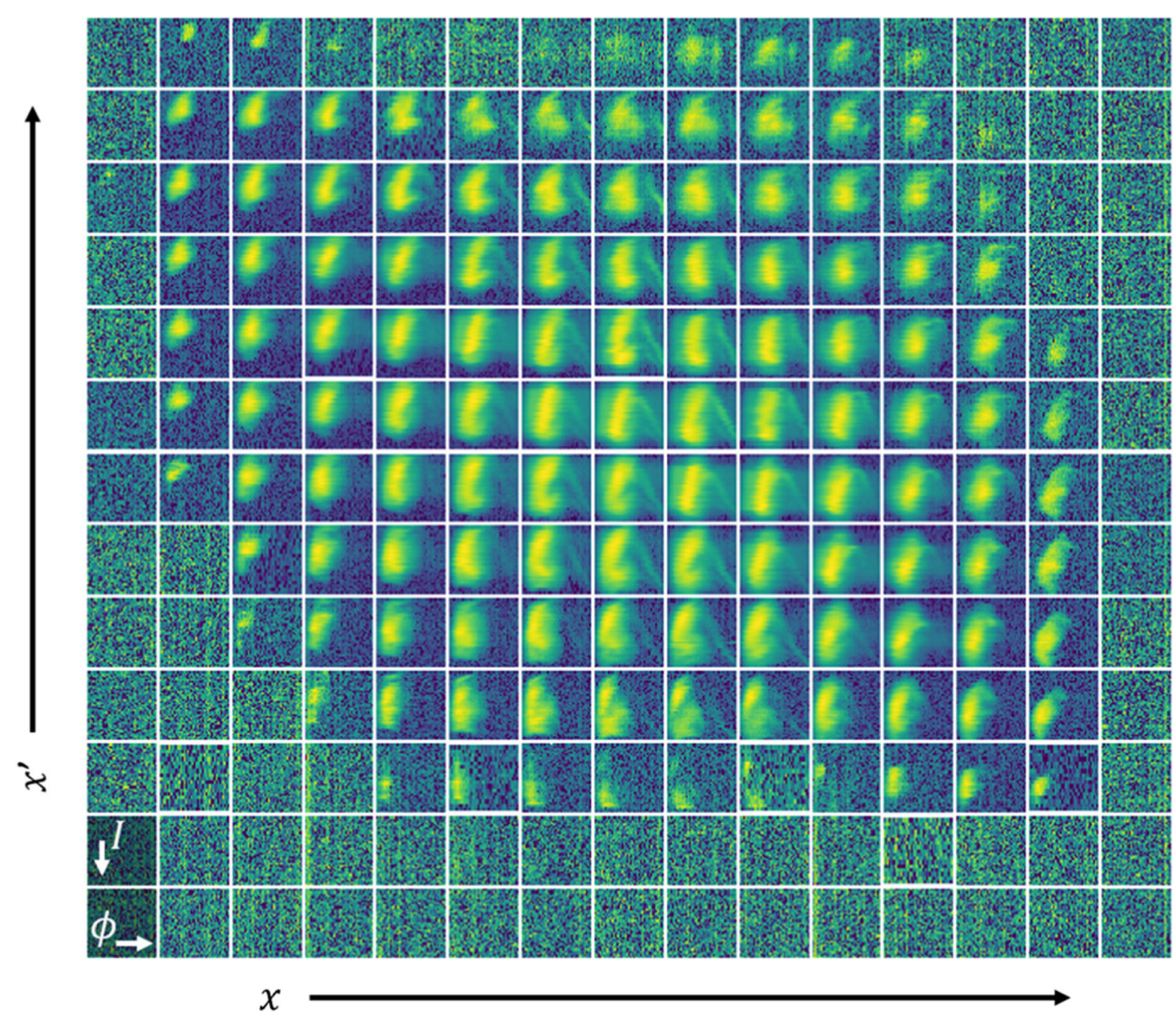

(a)

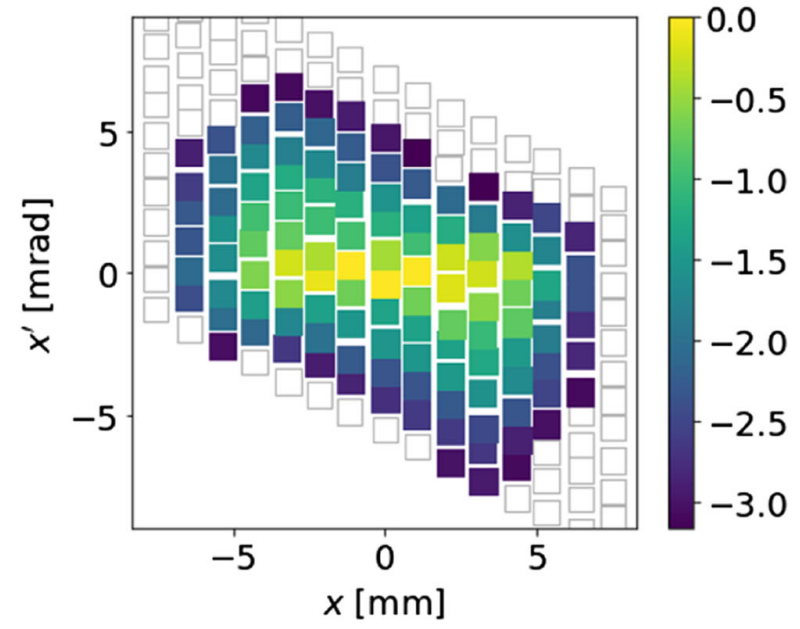

(b)

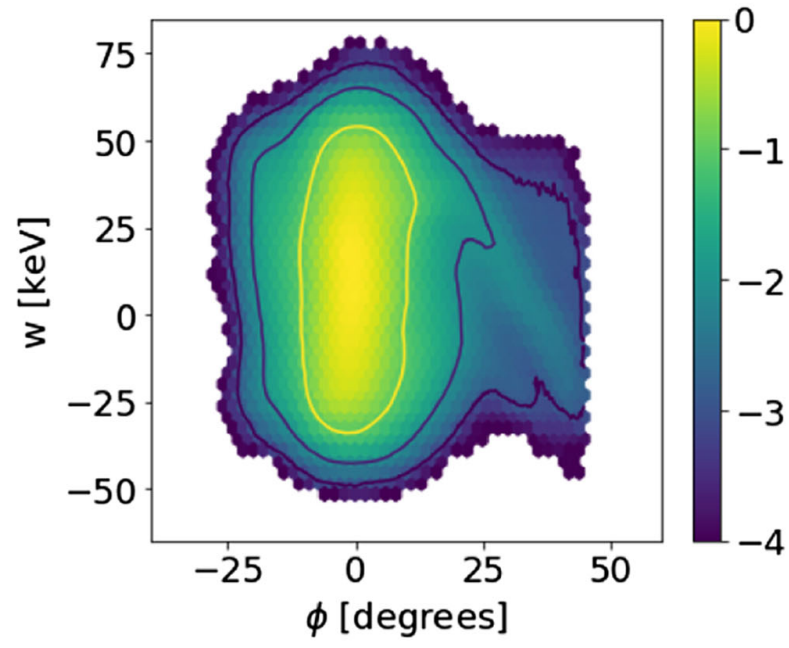

(c)

FIG. 12. Data from a 4D scan with BSM wire positioned for peak signal strength near the core of the beam. (a) Minimally-processed data from the 4D scan of $\left.\hat{f}\left(x, x^{\prime}, \phi, I\right)\right|_{\tilde{y}_{2}}$. Energy $w$ can be calculated through matrix transformation of $x, x^{\prime}$ and dipole current $I$. Each frame shows a partial projection $\left.\hat{f}(\phi, I)\right|_{\tilde{x}, \tilde{x}^{\prime}}$, with vertical axis $I$ and horizontal axis $\phi$. The axis limits are held fixed for all subplots, but the color scale is not. Each sub-frame corresponds to a different location in $x, x^{\prime}$. Color is signal strength in logarithmic scale. Data has been cleaned of spurious signals and averaged. (b) Image of the 4D scan along the $x, x^{\prime}$ axes, which are referenced to the location of the first vertical slit. The color of each point is the integrated signal in the $\phi, w$ dimensions. Points with no signal above $10^{-3.22}$ threshold are not filled. The intensity scale is logarithmic. (c) Longitudinal phase space $\left.\hat{f}(\phi, w)\right|_{\tilde{y}_{2}}$ generated by integrating frames in (a) over the horizontal coordinates, Contour levels are shown at $0.1 \%, 1 \%$ and $10 \%$ of peak. 
to be $80 \mathrm{kV}$ for the measurements reported here, while simulations apply $83 \mathrm{kV}$. The measured and simulated emittance curves are fairly flat between these two values.

The measured emittance is significantly lower than expected near design voltage. The source of discrepancy is not known. There is some uncertainty in the initial current, but as visualized in Figure 11(a) this is a small effect. The form of the LEBT distribution is more significant, as the simulation case with initial waterbag distribution overlaps with measurement for low voltages. The gradual emittance increase at high RFQ voltages is not reproduced in simulation. The sharp increase at low voltages, which coincides with formation of a low-energy tail, is in good agreement.

\section{B. Integrated 4D emittance}

As described above, the technique for measuring longitudinal emittance requires selecting three slices in the transverse dimensions. In order to reconstruct the integrated emittance $\left.\hat{f}(\phi, w)\right|_{\tilde{y}_{2}}$, a 4D scan over variables $\left(x, x^{\prime}, \phi, w\right)$ is performed.

The 4D scan is programmed as a nested loop of the three actuators that select the three dimensions $\left(x, x^{\prime}, w\right)$. These are the first two vertical slits and the dipole current: $\left(x_{1}, x_{2}, I\right)$. The slice $\tilde{y}_{2}$ was chosen to give the peak signal strength at the BSM, which corresponds to the BSM wire bisecting the core of the beam. Figure 12(b) illustrates the resolution of the 4D scan in transverse phase space by plotting the partial projection $\left.\hat{f}\left(x, x^{\prime}\right)\right|_{\tilde{y}_{2}}$. The minimally processed 4D scan data is shown in Fig. 12(a). Each subplot is the phase space $\left.\hat{f}(\phi, w)\right|_{\tilde{x}, \tilde{x}^{\prime}, \tilde{y}_{2}}$ for a point in $x, x^{\prime}, y_{2}$ space, corresponding with the scatter points in Figure 12(b).

Figure 12(c) shows the same data integrated over $x$ and $x^{\prime}$ to construct the 1D partial projection, $\left.\hat{f}(\phi, w)\right|_{\tilde{y}_{2}}$. In addition to integration, significant processing of the data has been done, including thresholding, correcting for variation in microchannel plate response and slow drifts in phase and RFQ output current. The output current over the 15.3 hour scan duration was on average $20.5 \pm 0.1 \mathrm{~mA}$. The emittance of the 1D slice with $1 \%$ threshold is $121-20 \pm 12 \mathrm{deg} \mathrm{keV}$. This can be compared to the emittance for the central frame only, $126-22 \pm 14$ deg keV. As expected from realistic simulations (Fig. 3, Table IV), the emittance of a 3D core slice is very close to the emittance of the lower-dimensional 1D slice.

\section{Dependence on vertical slice}

The integrated 4D emitttance shown in Fig. 12 is still a partial projection, due to the intersection of the BSM wire with the vertical phase space. Dependence on the BSM wire location is measured by repeating the $4 \mathrm{D}$ scan procedure at different wire positions. Figure 13 shows the resulting rms

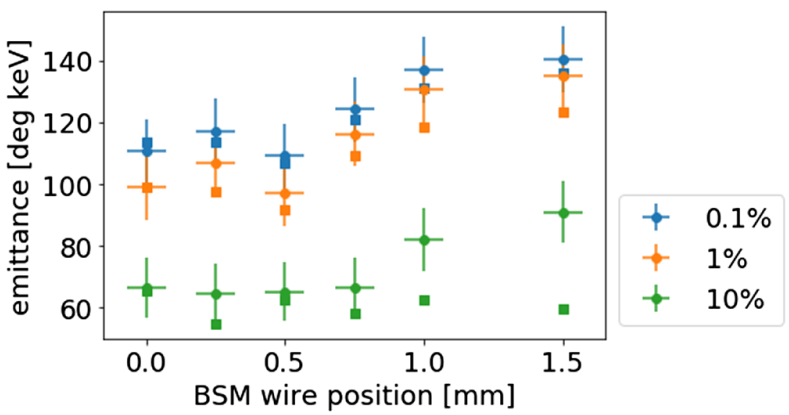

FIG. 13. Dependence of longitudinal emittance on position of BSM wire. The rms emittance is calculated for three threshold levels for both the 1D slice $\left.\hat{f}(\phi, w)\right|_{\tilde{y}_{2}}$ (shown with error bars) as well as 3D slice $\left.\hat{f}(\phi, w)\right|_{\tilde{x}, \tilde{x}^{\prime}, \tilde{y}_{2}}$ (square points without error bars) at fixed location $\tilde{x}, \tilde{x}^{\prime} \sim 0, y_{2} \propto \mathrm{BSM}$ wire.

slice emittances for $\left.\hat{f}(\phi, w)\right|_{\tilde{y}_{2}}$ and $\left.\hat{f}(\phi, w)\right|_{\tilde{x}, \tilde{x}^{\prime}, \tilde{y}_{2}}$ versus wire position. BSM wire position is reported in terms of distance from beam center at the plane of the wire. The center is determined to be the BSM wire position with the highest recorded signal intensity, with a precision $\pm 0.25 \mathrm{~mm}$.

There is a clear trend of lower emittances in the 1D $y_{2}$ slice near the core compared to edge slices. For the $0.1 \%$ and $1 \%$ emittances, the emittance of the high-dimensional slice $\left.\hat{f}(\phi, w)\right|_{\tilde{x}, \tilde{x}^{\prime}, \tilde{y}_{2}}$ is within error bars of the single-slice $\left.\hat{f}(\phi, w)\right|_{\tilde{y}_{2}}$ emittance, reinforcing the observation that the core slice emittance has low dependence on slice dimensionality. This breaks down at the $10 \%$ threshold, where the high-dimensional 3D slice emittance is noticeably lower than the 1D slice and has a flat dependence on transverse position.

\section{COMPARISON TO SIMULATION}

In general, the measured rms emittance was 20\%-30\% lower than simulated, a discrepancy that exceeds the $1 \sigma$ uncertainty. This was illustrated above in the comparison of RFQ voltage dependence (Fig. 11). More detailed comparison to the expected distribution, shown in Tables VI

TABLE VI. Comparison of simulated (expected values) and measured emittances for partially projected phase space $\left.\hat{f}(\phi, w)\right|_{\tilde{x}, \tilde{x}^{\prime}, \tilde{y}_{2}=0}$. Expected distribution values have slice width twice that of physical slit and wire width, for improved particle statistics. Comparison is not made for $0.1 \%$ threshold due to low number of particles in 3D slice.

\begin{tabular}{lccc}
\hline \hline Threshold & Quantity & Measured & Expected \\
\hline $0.1 \%$ & $\epsilon_{z}$ [deg.-keV] & $147-25 \pm 13$ & $\ldots$ \\
$1 \%$ & $\epsilon_{z}$ [deg.-keV] & $126-21 \pm 13$ & 131 \\
$10 \%$ & $\epsilon_{z}[$ deg.-keV] & $81-17 \pm 11$ & 96 \\
$1 \%$ & rms $\phi$ [deg.] & $5.6-0.7 \pm 0.6$ & 5.0 \\
$1 \%$ & rms $w$ [keV] & $22.4-1.1 \pm 0.4$ & 26.0 \\
\hline \hline
\end{tabular}


TABLE VII. Comparison of simulated (expected values) and measured emittances for partially projected phase space $\left.\hat{f}(\phi, w)\right|_{\tilde{y}_{2}=0}$. The slice applied to the simulated (expected) distribution is comparable to BSM wire width.

\begin{tabular}{lccc}
\hline \hline Threshold & Quantity & Measured & Expected \\
\hline $0.1 \%$ & $\epsilon_{z}$ [deg.-keV] & $133-23 \pm 12$ & 122 \\
$1 \%$ & $\epsilon_{z}$ [deg.-keV] & $119-20 \pm 12$ & 114 \\
$10 \%$ & $\epsilon_{z}$ [deg.-keV] & $86-18 \pm 11$ & 86 \\
$1 \%$ & rms $\phi$ [deg.] & $5.6-0.8 \pm 0.6$ & 5.1 \\
$1 \%$ & rms $w$ [keV] & $21.0-1.0 \pm 0.4$ & 22.7 \\
\hline \hline
\end{tabular}

and VII, shows that this discrepancy persists at all threshold levels. This is visualized further in Fig. 14.

The rms emittance has a weak dependence on dimensionality, as there is little difference between 3D and 1D slice emittances. Dependence of the 3D rms slice emittance on the slice center can be used to probe internal structure. Figure 15 shows the dependence of 3D slice emittance on the three transverse coordinates $x, x^{\prime}, y_{2}$. Simulations predict a flat dependence on $x^{\prime}$ and $y_{2}$ and a monotonic

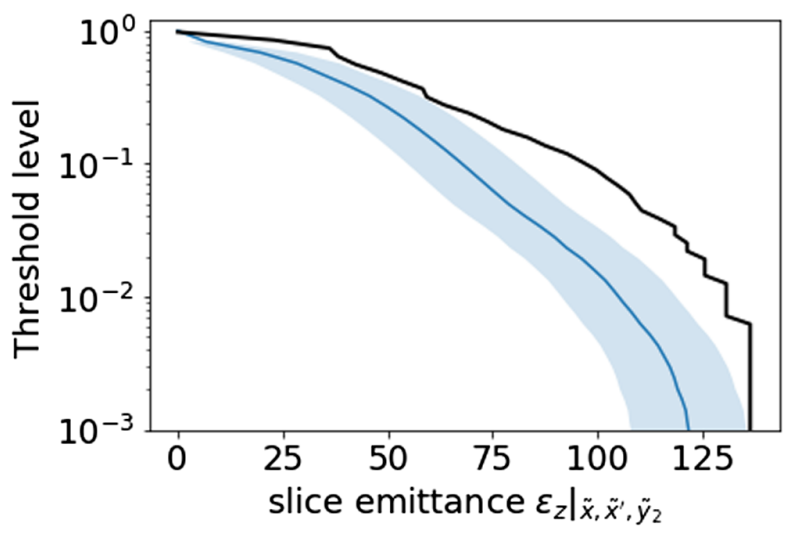

FIG. 14. Dependence of measured (blue curve, with uncertainty interval) and simulated slice emittances (black curve) for slice $\tilde{x}, \tilde{x}^{\prime}, \tilde{y}_{2}=0$. The correction to point-spread error is applied to measured values.
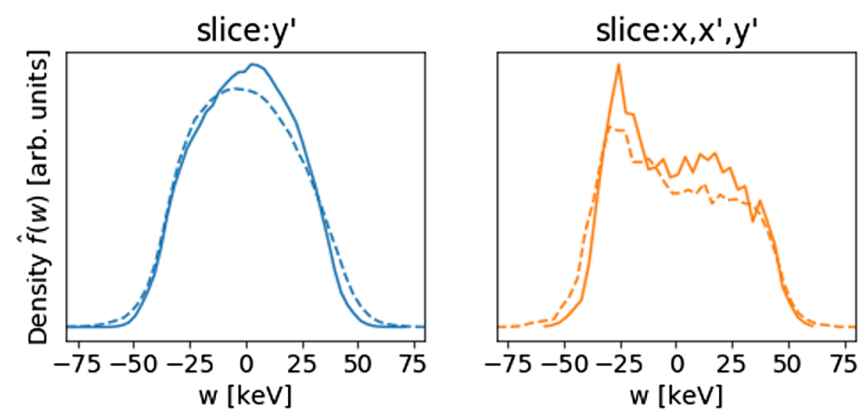

FIG. 16. Comparison of partially projected energy profile for measurement (solid lines) and simulation (dashed lines) for 1D (left plot) and $3 \mathrm{D}$ (right plot) slices. Here, $\vec{y}_{2} \approx \vec{y}^{\prime}$ is approximated. The simulated bunch has MEBT current $27 \mathrm{~mA}$. Measurement is for $20.5 \mathrm{~mA}$ in the MEBT.

decreasing dependence on $x$. In measurement, the dependence on $y_{2}$ was shown to be convex, while the behavior along $x$ and $x^{\prime}$ follows the expected trends.

Figure 16 compares the measured and simulated 1D and $3 \mathrm{D}$ partial energy projections. The general shape is reproduced, particularly for the 3D slice profile which is lop-sided with a maximum on the low-energy side. The energy width is very sensitive to beam current, as this grows during the first few centimeters of transport during debunching. It is difficult to compare to the expected bunch, as at the design current the energy width is much broader. For Fig. 16, the current of the expected bunch is artificially decreased at the RFQ exit from 41 to $27 \mathrm{~mA}$. The result matches the measured energy profile at $20 \mathrm{~mA}$. This effect cannot explain the emittance discrepancy, as this process occurs without emittance growth.

Finally, one prominent feature not recreated in simulation is the tail trailing the main bunch. This feature is very visible, for example in Figs. 7 and 12(c). The tail is included in the $1 \%$ and $0.1 \%$ emittance calculations, but excluded when a $10 \%$ threshold is applied. The tail signal diminishes with RFQ amplitude; it is interpreted as an artifact of operating at slightly below-optimal RFQ voltage.
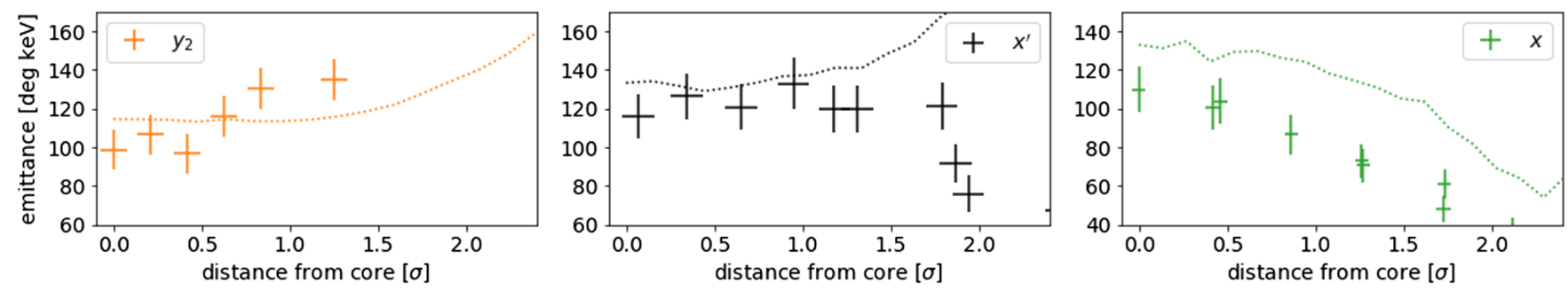

FIG. 15. Dependence of $1 \%$ rms emittance on position of slices in the transverse coordinates, compared against simulation of the expected distribution (dotted lines) The left-most plot shows emittances for a 1D slice $\tilde{y}_{2}$. The measured points are the same as shown in Figure 13. The remaining two plots show emittance for the 3D slice $\tilde{x}, \tilde{x}^{\prime}, \tilde{y}_{2}$. In the middle, the center of slice $\tilde{x}^{\prime}$ is varied while keeping $\tilde{x}, \tilde{y}_{2}=0$. On the right, the center of slice $\tilde{x}$ is varied for $\tilde{x}^{\prime}, \tilde{y}_{2}=0$. Distance from core is normalized to rms beam width, to account for difference in simulated and measured transverse beam size. 


\section{DISCUSSION}

The broader aim of this research is to improve accuracy of initial distributions to enable loss-level predictions. As illustrated here, the output bunch from the RFQ includes significant interplane coupling, which was previously shown to be driven by space charge [9]. Therefore, at high intensity the typical characterization of $2 \mathrm{D}$ phase space projections provides insufficient information for bunch reconstruction. A goal of ongoing studies is to generate fully correlated bunches based entirely on measurement. Until this goal is realized, end-to-end simulations are the best source of representative 6D distributions.

Nonlinear dynamics within the RFQ may increase sensitivity to errors in both external and self-fields, which in turn will limit the accuracy of the simulated bunch. This article reports a high-dimensional characterization of the longitudinal phase space in a test-stand mock-up of the SNS front-end and compares the results with parameters of an expected bunch from simulation. The expected bunch is generated by propagating a measurement-based LEBT distribution through the RFQ assuming design parameters.

Measured rms longitudinal emittances were lower than in simulation, but the 20\%-30\% margin is consistent with errors typically seen in RFQ model-measurement comparisons (e.g., [26,27]), and is an improvement on the $80 \%$ discrepancy reported for independent measurements in the SNS MEBT [28]. Qualitative agreement in high-dimensional views suggests that space charge forces are properly resolved, consistent with earlier conclusions that the cylindrically symmetric solver in PARMTEQ is sufficient [29].

The benchmark of rms emittances to within $30 \%$ is encouraging when considering the many diagnostic blind spots. First of all, the LEBT distribution is not well known. The realistic case adopted here is based on measurements from an old source at higher current and the 4D transverse distribution is reconstructed from $2 \mathrm{D}$ projections. As shown here, the form of the initial 4D distribution can affect the output emittance on level with the observed discrepancy.

Second, the output beam current is much lower than expected in the ideal system, which predicts $82 \%$ transmission for $50 \mathrm{~mA}$ input. During these measurements, the ion source produced approximately $40 \mathrm{~mA}$ with $60 \%$ transmission through the LEBT/RFQ system. The low transmission could reflect field errors within the RFQ, plausible due to past detuning events and repairs. Alternatively, errors in the LEBT could lead to reduced transmission. Improved agreement may be possible assuming a misaligned beam, as this is known to enhance losses in the RFQ through a combination of acceptance and image charge effects and may also influence emittance [29].

A unique opportunity exists to repeat this characterization for three versions of the SNS RFQ with identical physics design. The measurements reported here characterize RFQ-1, the original SNS RFQ [30]. RFQ-3 is expected to be delivered in 2021 and will be commissioned at the BTF. Afterwards, it will be switched with the current SNS production RFQ-2. Comparison of the three RFQs will provide insight into origins of the reported discrepancy, particularly with respect to possible field errors in RFQ-1.

Improvements to measurement techniques will enhance future studies. As discussed, the vane voltage is not independently measured and is likely nonoptimal, leading to the visible tail in phase space. This will be addressed via a nonintrusive bremsstrahlung voltage diagnostic [31]. The other major limitation is phase resolution. Although this error is suppressed in virtual slit reconstruction, it doubles measurement time and reduces signal-to-noise. Installation of a narrower energy slit is planned. Future upgrades will consider addition of a rebuncher cavity upstream of the $\mathrm{BSM}$ to reduce the linear $\phi-w$ correlation.

As beam halo mitigation becomes a more pressing concern for high-intensity accelerators, the need for predictive accelerator models will grow. While end-to-end simulation is a powerful tool for generating fully-correlated distributions, discrepancy in rms parameters precludes halo-level benchmarking. Characterization of the fullyformed bunch downstream of the RFQ is a better strategy. The most straightforward approach is full and direct $6 \mathrm{D}$ measurement. In the future, measurement burden may be reduced through synthesis of simulated and measured information. In prior work, rescaling simulated coordinates for rms equivalence was not sufficient [6]. Therefore, progress also requires new tools and metrics for highdimensional information.

\section{ACKNOWLEDGMENTS}

The authors acknowledge the contributions of Brandon Cathey, who not only authored the initial high dimensional beam study [9] but also the data collection software used here. We also thank Andrei Shishlo for useful discussion and assistance with the PyORBIT code. We are also very grateful for the support of SNS operations during long study periods. This manuscript has been authored by UTBattelle, LLC under Contract No. DE-AC05-00OR22725 with the U.S. Department of Energy. This research used resources at the Spallation Neutron Source, a DOE Office of Science User Facility operated by the Oak Ridge National Laboratory. The United States Government retains and the publisher, by accepting the article for publication, acknowledges that the United States Government retains a non-exclusive, paid-up, irrevocable, world-wide license to publish or reproduce the published form of this manuscript, or allow others to do so, for United States Government purposes. The Department of Energy will provide public access to these results of federally sponsored research in accordance with the DOE Public Access Plan [32]. 


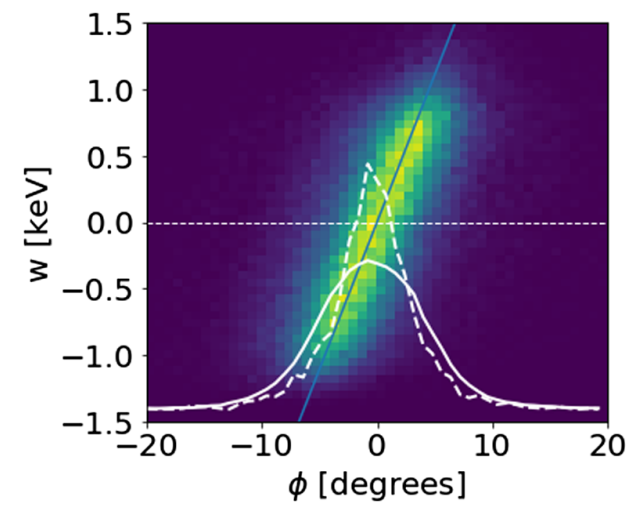

FIG. 17. $f(\phi, w)$ phase space immediately after selection at the energy slit. The solid white line plots the projected phase distribution $f(\phi)$. The dashed line shows the partial phase distribution $\left.\hat{f}(\phi)\right|_{w=0}$ (along the thin horizontal dashed line). This distribution is generated through PyORBIT simulation.

\section{APPENDIX A: SOURCES OF ERROR}

There are many potential sources of error. These can be separated into four categories by origin, ordered by effect on the measurement: (1) Resolution in energy and phase, determined by the physical width of the three slits as well as the phase resolution of the BSM. (2) Calibration errors, which are applied in the calculation of energy and phase coordinates. This uncertainty is determined by the variance of a linear least squares fit of the calibration data. (3) Model geometry, including uncertainty in path length and strength of magnetic elements, used in the calculation of energy. This includes uncertainty in machine readbacks such as slit position and dipole current. (4) Machine variation, encompassing both slow drifts and jitter. The largest source of error is due to the resolution of the measurement. The finite slit width create point-spread in both the energy and phase dimensions that result in systematic over-estimation of rms parameters. While the total point-spread is the combination of the three vertical slits, BSM wire and internal BSM electron focusing, the largest term is the width of the third (energy) slit. The energy point-spread is relatively small: $0.6 \mathrm{keV}$ compared to the rms width $23 \mathrm{keV}$ of the expected distribution.

However, for the phase coordinate the relative error is much larger. The main contribution to phase spread comes from two sources: the finite width of the energy slice and the electron optics in the BSM. The point-spread of the $\mathrm{BSM}$ can be directly measured by disabling the BSM RF deflector and recording the image of the BSM wire. The measured rms width of the internal BSM point-spread is $0.9^{\circ}$.

The energy spread contributes to phase spread at the BSM through time-of-flight. For a collection of particles with rms energy spread $0.6 \mathrm{keV}$ originating at the same phase in the plane of the energy slit, the phase spread at the BSM will be $0.5^{\circ}$. However, there is an additional, larger point spread effect due to the fact that at the energy slit the bunch is already highly correlated. Therefore, the projected phase width is significantly wider than the phase width of a monoenergetic slice. This effect is illustrated in Fig. 17, which is generated through PyORBIT simulation of the expected distribution to the energy slit. The $100 \% \mathrm{rms}$ phase width is $5.8^{\circ}$, but through projection of the correlated phase space, the apparent width increases to $6.6^{\circ}$. Assuming Gaussian phase distribution and point spread function, the rms width of the point-spread from correlation is estimated to be $3.5^{\circ}$. Adding the three sources of phase spread in quadrature, the total rms point-spread is $3.3^{\circ}$, roughly half the expected width.

Calibration errors make the next largest contribution and dominate the calculation of systematic uncertainty. This includes calibration of dipole strength $(-1.009 \pm 0.006$ $\mathrm{mm} / \mathrm{A}$ measured response of the beam at the energy slit) and conversion of BSM camera pixels to arrival phase $(0.167 \pm 0.008$ degrees/pixel). These uncertainties grow linearly with distance from the central phase and nominal dipole current. At the rms width of the expected distribution $w=23 \mathrm{keV}, \delta w= \pm 0.4 \mathrm{keV}$ and at $\phi=5.6^{\circ}, \delta \phi= \pm 0.6^{\circ}$.

Finally, uncertainty in the model geometry used to calculate beam energy has a negligible effect on calculated errors. Variations in the BTF beam and measured signal also have a negligible contribution. The effect of jitter (item 5) is reduced through averaging, and the overall statistical uncertainty is low. Slow variations, including drifts in phase, RFQ output current (Fig. 18) and BSM microchannel plate response, are corrected before emittance is calculated.

Assuming that the calibration errors are independent, they can be summed in quadrature to estimate the

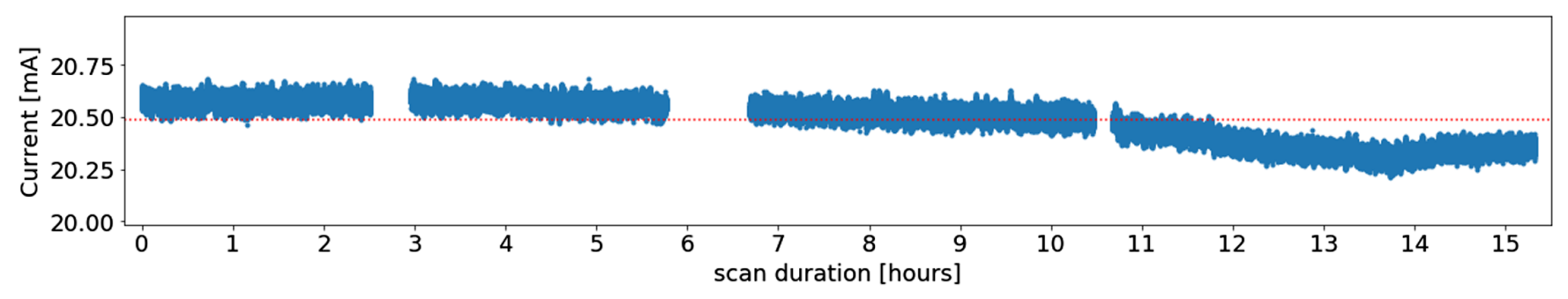

FIG. 18. Output at beam current monitor during collection of results shown above. Red dashed line indicates average over scan of $20.48 \mathrm{~mA}$. Pauses are due to routine lock-outs of BTF facility. 


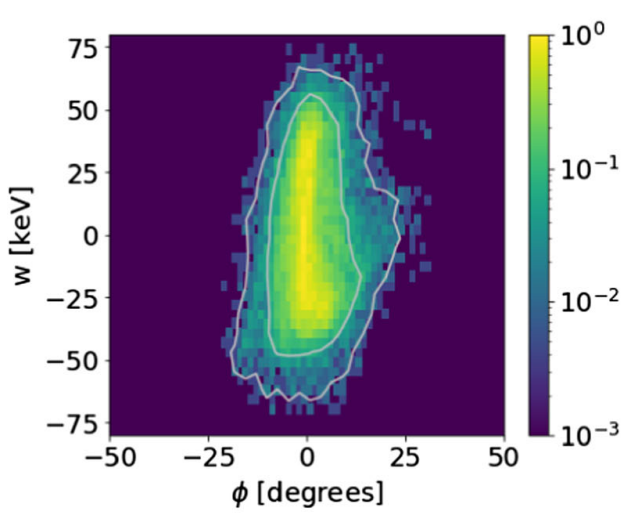

(a)

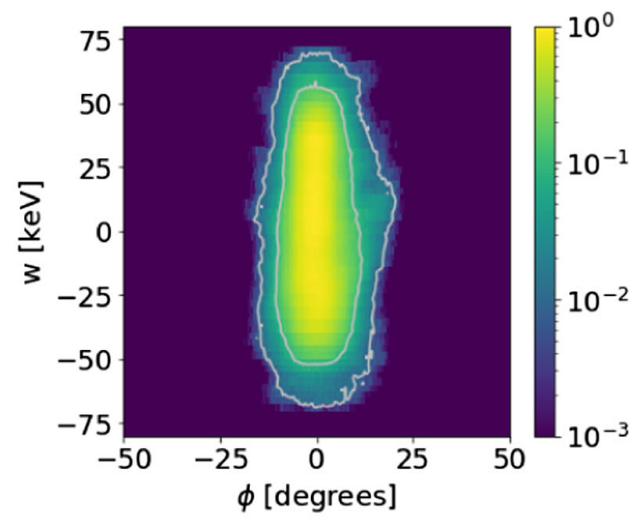

(b)

FIG. 19. Comparison between $\left.\hat{f}(\phi, w)\right|_{\tilde{x}, \tilde{x}}$ at (a) the first slit and (b) after reconstruction through the emittance apparatus with finite slit widths. $1 \%$ and $10 \%$ contour lines are drawn.

uncertainty in the measured emittance. The same can be done for the systematic error of the point spread function. In the approximation $\epsilon_{z} \approx \Delta \phi \Delta w$, the error propagates as

$$
\left(\frac{\delta \epsilon_{z}}{\left\langle\epsilon_{z}^{2}\right\rangle}\right)^{2} \approx\left(\frac{\delta \phi}{\left\langle\phi^{2}\right\rangle}\right)^{2}+\left(\frac{\delta w}{\left\langle w^{2}\right\rangle}\right)^{2}
$$

\section{APPENDIX B: SIMULATED ESTIMATE OF POINT SPREAD ERROR}

The analytic error estimate is based on the assumption that the $\hat{f}(\phi, w)$ distribution and point spread functions are Gaussian, which is an incorrect assumption. In order to more carefully estimate the systematic error due to finite slit widths, the longitudinal emittance measurement was reproduced with PyORBIT simulation. The expected distribution from the RFQ is tracked to the location of the BSM wire, with slit apertures applied as in measurement. The first two vertical slits are centered at $\tilde{x}=0 \pm 0.1 \mathrm{~mm}$, $\tilde{x}^{\prime}=0 \pm 0.2 \mathrm{mrad}$. The simulation is repeated for different positions of the third (energy) slit, at a spacing of $\Delta w=0.25 \mathrm{keV}$. Just as in measurement, the emittance $\hat{f}(\phi, w)$ is reconstructed by combining these phase distributions. The selection of vertical phase space at the BSM wire is not included, as it should have a negligible effect on the point spread error.

The distribution is generated through RFQ simulation with $5 \mathrm{e} 6$ particles. This large number is chosen to maintain good statistics after the slit apertures. At each $0.2 \mathrm{~mm}$ slit aperture, only about $2 \%$ of particles pass through. After each of the first two slits, the distribution is smoothed and resampled back to $5 \mathrm{e} 6$ particles. The re-sampling results in slight artificial growth of the longitudinal emittance at each slit (about $1 \%$ at each). This is smaller than the point spread effect.

The reconstructed distribution is compared to the longitudinal distribution in the plane of the first vertical slit in Figure 19. No significant space-charge influenced evolution of the longitudinal emittance is expected between this location and the BSM. The broadening of the distribution due to finite slit width is apparent, particularly in the phase width.

\section{APPENDIX C: SUBSLIT RESOLUTION WITH VIRTUAL SLIT}

As established, the largest error in the emittance measurement is due to the point spread associated with the finite width of the energy slit. However, it is possible to obtain a higher resolution that overcomes the physical limitations of the existing apparatus, without the need to manufacture and install narrower slits. This is done by creating a virtual slit from two phase profiles separated by a differential step in slit position. The step size must be smaller than the physical slit width for enhanced resolution.

As in the typical emittance measurements, the dipole current is varied rather than actuating the energy slit. This is particularly beneficial for the virtual slit measurements, as the dipole current can be set with higher precision ( $\pm 0.005 \mathrm{~A}$, equivalent to $0.005 \mathrm{~mm}$ response at energy

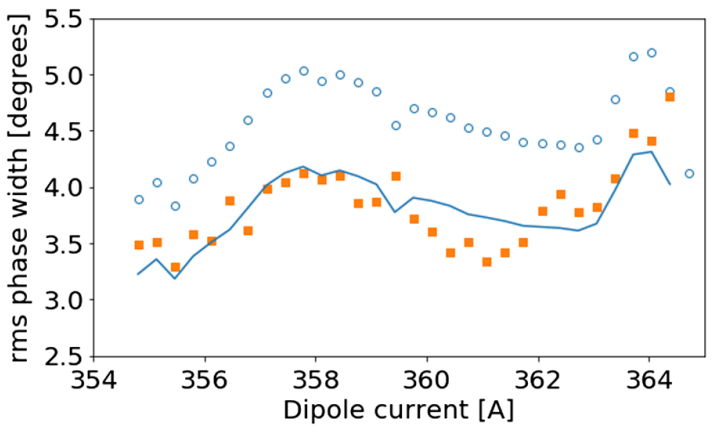

FIG. 20. Comparison of rms phase width at $1 \%$ threshold. Solid orange points are phase width measured with virtual slit technique. Open blue points are result of "typical" measurement. The solid blue line is the same measurement with $83 \%$ correction factor applied. 
TABLE VIII. Comparison of virtual slit reconstructed emittance with "typical" measurement at $1 \%$ threshold.

\begin{tabular}{lccc}
\hline \hline Quantity & Typical & Virtual slit & Uncertainty \\
\hline $\operatorname{rms} \epsilon_{\phi}[\mathrm{deg} \mathrm{keV}]$ & 120 & 102 & 13 \\
$\operatorname{rms} \phi$ & $5.4^{\circ}$ & $4.7^{\circ}$ & $0.6^{\circ}$ \\
$\operatorname{rms~} w[\mathrm{keV}]$ & 23.1 & 22.4 & 0.4 \\
\hline \hline
\end{tabular}

slit) than the slit actuator position $( \pm 0.02 \mathrm{~mm})$. A virtual slit spacing of $0.05 \mathrm{~A}(0.05 \mathrm{~mm})$ was found to be sufficient in both simulation and measurement. In application of the virtual slit reconstruction on simulated data, the recovered phase width plateaued for slit separations $\leq 0.07 \mathrm{~mm}$ was within $5 \%$ of the "base truth" phase width. In measurement, the recovered phase width appears to plateau at 0.05 A separation.

Figure 20 compares the measured rms phase widths at $1 \%$ threshold for a range of energies (plotted as dipole current). Phase width is calculated both with and without application of the virtual slit technique. The reconstructed phase profiles are significantly more noisy, but on average (for all profiles measured in this dataset), the reconstructed width is equal to $83 \%$ of the measured width without correction (shown in the figure as a solid line).

Table VIII compares rms for a "typical" phase space measurement with the reconstruction using the virtual slit technique. Note that there is still uncorrected point-spread from the internal BSM optics, but at rms $0.9^{\circ}$ this leads to a much smaller error than from the energy slit.

[1] S. Henderson, Accelerator and target technology for accelerator driven transmutation and energy production, Technical Report (2010).

[2] A. V. Fedotov, Mechanisms of halo formation, AIP Conf. Proc. 3, 610 (2003).

[3] R. L. Gluckstern, Analytic Model for Halo Formation in High Current Ion Linacs, Phys. Rev. Lett. 73, 1247 (1994).

[4] N. Chauvin, O. Delferrière, R. Duperrier, R. Gobin, P. A. Nghiem, and D. Uriot, Transport of intense ion beams and space charge compensation issues in low energy beam lines (invited), Rev. Sci. Instrum. 83, 02B320 (2012).

[5] C. K. Allen, K. C. D. Chan, P. L. Colestock, K. R. Crandall, R. W. Garnett, J. D. Gilpatrick, W. Lysenko, J. Qiang, J. D. Schneider, M. E. Schulze, R. L. Sheffield, H. V. Smith, and T. P. Wangler, Beam-Halo Measurements in High-Current Proton Beams, Phys. Rev. Lett. 89, 214802 (2002).

[6] J. Qiang, P. L. Colestock, D. Gilpatrick, H. V. Smith, T. P. Wangler, and M.E. Schulze, Macroparticle simulation studies of a proton beam halo experiment, Phys. Rev. Accel. Beams 5, 124201 (2002).

[7] L. Groening, W. Barth, W. Bayer, G. Clemente, L. Dahl, P. Forck, P. Gerhard, I. Hofmann, G. Riehl, S. Yaramyshev, D. Jeon, and D. Uriot, Benchmarking of measurement and simulation of transverse rms-emittance growth, Phys. Rev. Accel. Beams 11, 094201 (2008).

[8] P. K. Roy, C.E. Taylor, C. Pillai, and Y. K. Batygin, Comparison of profile measurements and TRANSPORT beam envelope predictions along the 80-m LANSCE pRad beamline, J. Phys. Conf. Ser. 1067, 062002 (2018).

[9] B. Cathey, S. Cousineau, A. Aleksandrov, and A. Zhukov, First Six Dimensional Phase Space Measurement of an Accelerator Beam, Phys. Rev. Lett. 121, 064804 (2018).

[10] T. F. Wang and L. Smith, Transverse-longitudinal coupling in intense beams, Part. Accel. 12, 247 (1982).

[11] A. Fedotov, R. Gluckstern, S. Kurennoy, and R. Ryne, Halo formation in three-dimensional bunches with various phase space distributions, Phys. Rev. Accel. Beams 2, 014201 (1999).

[12] A. W. Chao, R. Pitthan, T. Tajima, and D. Yeremian, Space charge dynamics of bright electron beams, Phys. Rev. Accel. Beams 6, 024021 (2003).

[13] T. P. Wangler, K. R. Crandall, R. Ryne, and T. S. Wang, Particle-core model for transverse dynamics of beam halo, Phys. Rev. Accel. Beams 1, 084201 (1998).

[14] A. Aleksandrov, Beam instrumentation for high power hadron beams, in Proc. 25th Part. Accel. Conf., edited by T. Satogata, C. Petit-Jean-Genaz, and V. Schaa (JACoW, Pasadena, CA, 2013), pp. 380-384.

[15] A. V. Feschenko, Methods and instrumentation for bunch shape measurements, in Proceedings of the 2001 Particle Accelerator Conference, Vol. 1 (IEEE, Chicago, IL, USA, 2001), pp. 517-521.

[16] Z. Zhang, S. Cousineau, A. Aleksandrov, A. Menshov, and A. Zhukov, Design and commissioning of the Beam Test Facility at the Spallation Neutron Source, Nucl. Instrum. Methods Phys. Res., Sect. A 949, 162826 (2020).

[17] A. Aleksandrov, S. Cousineau, K. Ruisard et al., First measurement of a $2.5 \mathrm{MeV}$ RFQ output emittance with 1 part-per-million dynamic range, Nucl. Instrum. Methods Phys. Res., Sect. A 987, 164829 (2021).

[18] K. R. Crandall and T. P. Wangler, PARMTEQ-A beamdynamics code fo the RFQ linear accelerator, AIP Conf. Proc. 177, 22 (1988).

[19] S. Henderson, W. Abraham, A. Aleksandrov, C. Allen, J. Alonso, D. Anderson, D. Arenius, T. Arthur, S. Assadi, J. Ayers, P. Bach, V. Badea, R. Battle, J. Beebe-Wang, B. Bergmann et al., The Spallation Neutron Source accelerator system design, Nucl. Instrum. Methods Phys. Res., Sect. A 763, 610 (2014).

[20] A. Ratti, R. Digennaro, R. A. Gough, M. Hoff, R. Keller, K. Kennedy, R. Macgill, J. Staples, S. Virostek, and Yourd, The design of a high current, high duty factor Rfq for the Sns, in Proc. EPAC 2000 (Vienna, Austria, 2000), pp. 495-497.

[21] A. Ratti, J. Ayers, L. Doolittle, R. Digennaro, R. A. Gough, M. Hoff, R. Keller, R. Macgill, J. Staples, R. Thomae, S. Virostek, R. Yourd, E. O. L. Berkeley, and A. Aleksandrov, The SNS RFQ commissioning, in Proceedings of the 21st International Linac Conference, Gyeongju, Korea, 2002 (Pohang Accelerator Laboratory, Pohang, Korea, 2002), p. 329. 
[22] I. M. Kapchinskij and V. V. Vladimirskij, Limitations of proton beam current in a strong focusing linear accelerator associated with beam space charge, in Proc. 9th Int. Conf. High Energy Accel., edited by L. Kowarski (CERN, Geneva, Switzerland, 1959) p. 274.

[23] A. Shishlo, S. Cousineau, J. Holmes, and T. Gorlov, The particle accelerator simulation code PyORBIT, Procedia Comput. Sci. 51, 1272 (2015).

[24] K. Ruisard and A. Aleksandrov, Rapid charge redistribution leading to core hollowing in a high-intensity ion beam, arXiv:2008.06561.

[25] K. Ruisard, A. Aleksandrov, and A. Shishlo, Virtual slit for improved resolution in longitudinal emittance measurement, in Proc. IBIC2020, Santos, Brazil (JACoW, 2020).

[26] J. B. Lallement, G. Bellodi, V. A. Dimov, A. M. Lombardi, and M. Y. Satri, Linac4 transverse and longitudinal emittance reconstruction in the presence of space charge, in Proceedings of the 20th International Linac Conference, LINAC-2000, Monterey, CA, 2000 (SLAC, Menlo Park, CA, 2000), pp. 913-915.

[27] G. Normand, M. Di Giacomo, R. Ferdinand, O. Kamalou, J. M. Lagniel, A. Savalle, and D. Uriot, SPIRAL2 RFQ bunch lengths and longitudinal emittance measurements, J. Phys. Conf. Ser. 1350, 012098 (2019).

[28] A. Shishlo, A. Aleksandrov, Y. Liu, and Z. Wang, Measuring longitudinal beam parameters in the low energy section of the Oak Ridge Spallation Neutron Source accelerator, Phys. Rev. Accel. Beams 21, 092803 (2018).

[29] F. W. Guy, Three-dimensional space charge and image charge effects in radio-frequency-quadrupole accelerators, in Proceedings of the IEEE 1991 Particle Accelerator Conference (APS Beams Physics) (IEEE, Piscataway NJ, 1991), p. 3032.

[30] A. Aleksandrov, M. Champion, M. Crofford, K. Ewald, Y. Kang, A. Menshov, M. Middendorf, S. Murray, T. Rosebery, R. Saethre, M. Stockli, A. Webster, R. Welton, A. Z. Ornl, and O. Ridge, Status of new $2.5 \mathrm{MeV}$ test facility at SNS, in Proc. LINAC2014 (JACoW, Geneva, Switzerland, 2014), pp. 1105-1107.

[31] A.P. Zhukov, SNS RFQ voltage measurements using X-ray spectrometer, Proc. 5th Int. Beam Instrum. Conf. IBIC 2016 (JACoW, Barcelona, Spain, 2016), p. 154.

[32] http://energy.gov/downloads/doe-public-access-plan. 